\title{
HIV-1 transmitted drug resistance-associated mutations and mutation co-variation in HIV-1 treatment-naïve MSM from 2011 to 2013 in Beijing, China
}

Yang Jiao ${ }^{1,2 \dagger}$, Shuming $\mathrm{Li}^{2 \dagger}$, Zhenpeng $\mathrm{Li}^{1}$, Zheng Zhang ${ }^{2}$, Jianhong Zhao ${ }^{2}, \mathrm{Li}_{\mathrm{Li}}{ }^{2}, \mathrm{Lijuan} \mathrm{Wang}^{2}$, Qianqian Yin', Yan Wang ${ }^{1}$, Zhaoli Zeng ${ }^{2}$, Yiming Shao ${ }^{1 *}$ and Liying $\mathrm{Ma}^{1^{*}}$

\begin{abstract}
Background: Transmitted drug resistance (TDR) is an important public health issue, because TDR-associated mutation may affect the outcome of antiretroviral treatment potentially or directly. Men who have sex with men (MSM) constitute a major risk group for HIV transmission. However, current reports are scarce on HIV TDR-associated mutations and their co-variation among MSM.

Methods: Blood samples from 262 newly diagnosed HIV-positive, antiretroviral therapy (ART)-naïve MSM, were collected from January 2011 and December 2013 in Beijing. The polymerase viral genes were sequenced to explore TDR-associated mutations and mutation co-variation.

Results: A total of 223 samples were sequenced and analyzed. Among them, HIV-1 CRF01_AE are accounted for 60.5\%, followed by CRF07_BC (27.8\%), subtype B (9.9\%), and others. Fifty-seven samples had at least one TDR-associated mutation, mainly including L10IN (6.3\%), A71L/TN (6.3\%), V179D/E (5.4\%), and V106I (2.7\%), with different distributions of TDR-associated mutations by different HIV-1 subtypes and by each year. Moreover, eight significant co-variation pairs were found between TDR-associated mutations (V179D/E) and seven overlapping polymorphisms in subtype CRF01_AE.

Conclusions: To date, this work consists the most comprehensive genetic characterization of HIV-1 TDR-associated mutations prevalent among MSM. It provides important information for understanding TDR and viral evolution among Chinese MSM, a population currently at particularly high risk of HIV transmission.
\end{abstract}

Keywords: HIV-1, MSM, Subtypes, Transmitted drug resistance-associated mutations, Co-variation

\section{Background}

The increasing availability of antiretroviral therapy (ART) worldwide has significantly reduced mortality and improved quality of life for HIV-infected patients. However, the therapeutic effect of ART is weakened by the emergence of drug-resistant mutant viruses. Transmission of

\footnotetext{
* Correspondence: yshao08@gmail.com; mal@chinaaids.cn

${ }^{\dagger}$ Equal contributors

${ }^{1}$ State Key Laboratory for Infection Disease Prevention and Control, National Center for AIDS/STD Control and Prevention (NCAIDS), Collaborative Innovation Center for Diagnosis and Treatment of Infectious Diseases, Chinese Center for Disease Control and Prevention (China CDC), Beijing 102206, China

Full list of author information is available at the end of the article
}

drug-resistant (TDR) strains to persons who are ARTnaive can compromise the effectiveness of treatment and limit antiretroviral regimens options. TDR consists $10 \sim$ $20 \%$ of new HIV-1 infections worldwide [1]. In resourcelimited countries such as China, where ART is provided to patients for free through the "Four Free One Care" policy [2], TDR against standard ART regimens threatens the stability of treatment programs and must be carefully monitored.

Several factors contribute to the occurrence of TDR, including frequency of exposure to non-treatment naïve viruses, ART regimen efficacy in the transmitting patient, rates of virologic suppression, and genetic diversity and 
replicative capacity of the viral strains in question. To our interest, evidence suggests that HIV-1 genetic diversity may influence the type and rate of resistance mutations that may eventually emerge upon drug exposure $[3,4]$. Previous studies showed a significant correlation between treatment-associated mutations and overlapping polymorphisms in the RT and PR viral genes [5]. We postulate that there may be co-variation between TDR-associated mutations and overlapping polymorphisms on treatment-naïve patients, which affect the transmission of drug resistance mutant viruses.

Previous evidence suggests that drug resistance mutations may result in significantly decreased replicative fitness and hence transmission efficacy [6]. Thus, we may expect persons at higher frequency of exposure to nontreatment-naïve viruses to be at higher risk of receiving drug resistant strains than those persons under lower frequency of exposure. In China, one such risk group with particularly high frequency of HIV-1 exposure is men who have sex with men (MSM). MSM in China typically have multiple sexual partners, low rates of condom usage, and low rates of HIV screening $[7,8]$. As well, they are a rapidly expanding high risk population for HIV transmission: the proportion of all reported cases of HIV infection in China with history of MSM sex has increased from $2.5 \%$ in 2006 to $13.7 \%$ in 2011 [9]. It is therefore important to examine TDR among MSM in China.

Our study focuses on Beijing, where the proportion of MSM carrying HIV has increased rapidly from $3.1 \%$ in 2002 [10] to $4.8 \%$ in 2006 [11]. The proportion of MSM among newly HIV diagnosed cases was $70.7 \%$ in 2012 [12], much higher than the corresponding rates in other cities in China [13-15]. Although several studies have reported TDR among ART-naïve MSM in Beijing [11,16,17], there lacks a systematic analysis on TDR rates over time and the co-variation of TDR-associated mutations. Thus, we performed a comprehensive genetic characterization of HIV-1 strains prevalent in MSM in Beijing from 2011 to 2013, analyzing TDR-associated mutations and mutation co-variation.

\section{Methods}

\section{Study patients}

A total of $262 \mathrm{HIV}-1$ positive individuals were randomly recruited from 2011 to 2013 at voluntary counseling and testing sites (VCT) in Beijing Chaoyang District Center for Disease Control and Prevention, following three criteria: having had history of MSM sex, being ART-naïve and newly diagnosed. This study was approved by the Institutional Research Ethics Community, China Chaoyang $\mathrm{CDC}$, and all subjects signed informed consent forms prior to blood collection. Epidemiological data was collected by trained interviewers. HIV-1 infection status was determined by an enzyme immunoassay (ELISA, Wantai, China) and confirmed by Western blot assay (HIV BLOT 2.2, MP Diagnostics, Singapore). Blood plasma was separated and stored at $-70^{\circ} \mathrm{C}$ prior to genetic analysis.

\section{HIV-1 RNA extraction, amplification and sequencing}

Viral RNA was extracted from $200 \mu \mathrm{l}$ EDTA-anticoagulated plasma using a QIAamp viral RNA kit (Qiagen Inc., Germany) according to the manufacturer's instructions. The HIV-1 pol gene (1,197 bp length), containing the fulllength protease (PR) gene and the first 300 codons of the reverse transcriptase (RT) gene, were amplified and sequenced, using an in-house drug resistance genotyping method as previously described [18]. The target sequence was amplified with One Step Reverse Transcription PCR reagents (Qiagen Inc., Germany) using primers listed in Table 1. Amplification steps were as follows: reverse transcription at $50^{\circ} \mathrm{C}$ for $30 \mathrm{~min}$, pre-denaturation at $94^{\circ} \mathrm{C}$ for $5 \mathrm{~min}, 30$ cycles of denaturation at $94^{\circ} \mathrm{C}$ for $30 \mathrm{~s}$, annealing at $55^{\circ} \mathrm{C}$ for $30 \mathrm{~s}$, extension at $72^{\circ} \mathrm{C}$ for $2.5 \mathrm{~min}$, and an additional extension at $72^{\circ} \mathrm{C}$ for $10 \mathrm{~min}$. Nested PCR was performed using Taq PCRmaster mix (Qiagen Inc., Germany) with primers in Table 1 . The cycling conditions were: predenaturation at $94^{\circ} \mathrm{C}$ for $5 \mathrm{~min}, 30$ cycles of denaturation at $94^{\circ} \mathrm{C}$ for $30 \mathrm{~s}$, annealing at $63^{\circ} \mathrm{C}$ for $30 \mathrm{~s}$, extension at $72^{\circ} \mathrm{C}$ $2.5 \mathrm{~min}$, and an additional extension at $72^{\circ} \mathrm{C}$ for $10 \mathrm{~min}$. PCR products were visualized by $1 \%$ agarose gel electrophoresis and sequenced using ABI 3730xl Automated DNA Analyzer (Applied Biosystems, Foster City, CA). Each step was carried out with negative controls.

\section{Phylogenetic analysis}

All assembled sequences were submitted to the Los Alamos National Laboratory HIV Sequence Database (http://www. hiv.lanl.gov/content/index) to determine HIV genotype, which were further confirmed by phylogenetic analysis using standard reference sequences representing subtypes A-D, F-H, J, K, CRF01_AE, CRF07_BC, and CRF08_BC (www.hiv.lanl.gov). DNA alignment was performed by the Clustal W method using MEGA5 [19], followed by manual adjustment. Phylogenetic analysis was also conducted with MEGA5 using neighbor-joining trees under a Kimura 2parameter model and tested by the bootstrap method with 1,000 replicates.

\section{Drug resistance analysis}

Sample pol gene sequences were compared to a consensus sequence using HIV db software (Stanford HIV Drug Resistance Database, http://hivdb.stanford.edu, version 7.0) to detect drug resistance mutations, including major and minor protease inhibitor (PI) resistance mutations, nucleoside reverse transcriptase inhibitor (NRTI), and non-nucleoside reverse transcriptase inhibitor (NNRTI) resistance mutations. 
Table 1 Primers used in the optimized in-house assay

\begin{tabular}{llll}
\hline Primer name & Sequence (5,-3,) & Location (based on HXB2) & Purpose \\
\hline MAW 26 & TTGGAATGGGGAAGGAAGGAC & 2028-2050 \\
RT21 & CTGTATTCTGCTATTAAGTCTTTGATGGG & $3509-3539$ & RT-PCR \\
PRO-1 & CAGAGCCAACAGCCCCACCA & $2147-2166$ & Nested PCR \\
RT20 & CTGCCAGTTCTAGCTCTGCTTC & $3441-3462$ & Nested PCR \\
MAW26-07BC & TGGAAATGTGGAAAGAAGGAC & $2028-2050$ & RT-PCR \\
RT21-07BC & CTGTATTTCAGCTATCAAGTCTTTGATGGG & $3509-3539$ & RT-PCR \\
PR01-07BC & CAGAGCCAACAGCCCCACCA & $2147-2166$ & Nested PCR \\
RT20-07BC & CTGCCAATTCTAATTCTGCTTC & $3441-3462$ & Nested PCR \\
MAW26-01AE & TGGAAATGTGGRAARGAAGGAC & $2028-2050$ & RT-PCR \\
RT21-01AE & GTAYTTCTGCTAYTAAGTCTTTGATGGG & $3511-3539$ & RT-PCR \\
PR01-01AE & CAGAGCCAWCAGCCCCACCA & $2147-2166$ & Nested PCR \\
RT20-01AE & CTGCCAAYTCTAATTCTGCTTC & $3441-3462$ & Nested PCR \\
\hline
\end{tabular}

\section{Co-variation analysis between TDR-associated mutations} and positively selected mutations

We analyzed co-variation between TDR-associated mutations and positive selected mutation using the CorMut package [20]. Briefly, the procedure was: positively selected mutations were identified using selection pressure $(\mathrm{Ka} / \mathrm{Ks}$ ratio) based method $[21,22]$, in which a $\mathrm{Ka} /$ Ks value of $>1$ indicates a positive selection. Log odds (LOD) confidence score was used to measure the significance of selection pressure (cut off $>=2$ ). The 05GX001 strain (subtype CRF01_AE) was used as a reference when performing the computation. The Jaccard similarity coefficient was used to measure the covariation between TDR mutations and positively selected mutations. Fisher's exact test was used to check the significance of co-variation. False discovery rate was controlled using the Benjamini and Hochberg procedure with a 0.2 cut-off. $P$ adjusted value $<0.2$ was considered statistical significance.

\section{Results}

\section{Patient characteristics}

Among $262 \mathrm{HIV}-1$ positive samples, the pol genes of 223 samples $(85.11 \%)$ were successfully amplified and sequenced. The mean age of the 223 patients was 30.3 (range: $17-64$ ). $75.3 \%$ of subjects were never married, $21.5 \%$ were married, and $3.1 \%$ were divorced or widowed. More than two-thirds of participants (69.5\%) had received college-level or higher education degree. The basic demographic characteristics are shown in Table 2.

\section{HIV-1 genetic characteristics}

Phylogenetic analysis of the amplified pol gene regions (1197 bp) showed that the samples were generally tightly clustered within their respective subtypes (Figure 1). Their genotype distribution was as follows: 135 cases
(60.5\%) were subtype CRF01_AE, 62 cases (27.8\%) were subtype CRF07_BC, 22 cases (9.9\%) were subtype B, two were CRF01B, one was CRF55_01B, and one was CRF08_BC (Table 2). There was no significant difference in HIV-1 subtype distributions between each year.

Table 2 Characteristic and genotypes of study subjects

\begin{tabular}{|c|c|c|c|c|}
\hline & \multirow[t]{2}{*}{ Total } & \multicolumn{3}{|c|}{ Year } \\
\hline & & 2011 & 2012 & 2013 \\
\hline Total & 223 & 21 & 126 & 76 \\
\hline \multicolumn{5}{|l|}{ Subtype } \\
\hline CRF01_AE & $135(60.53 \%)$ & $12(57.14 \%)$ & $80(63.49 \%)$ & $43(56.58 \%)$ \\
\hline CRF07_BC & $62(27.80 \%)$ & $7(33.33 \%)$ & $31(24.60 \%)$ & 24(31.58\%) \\
\hline B & $22(9.86 \%)$ & $2(9.53 \%)$ & $12(9.52 \%)$ & $8(10.53 \%)$ \\
\hline Others & $4(1.79 \%)$ & 0 & $3(2.38 \%)$ & $1(1.32 \%)$ \\
\hline \multicolumn{5}{|l|}{ Age } \\
\hline Mean & 30.79 & 27.76 & 31.35 & 30.71 \\
\hline$\leq 24$ & $48(21.52 \%)$ & $7(33.33 \%)$ & 23(18.26\%) & $18(23.68 \%)$ \\
\hline $25-34$ & $116(52.02 \%)$ & $11(52.38 \%)$ & $71(56.35 \%)$ & $34(44.74 \%)$ \\
\hline $35-44$ & $44(19.73 \%)$ & $3(14.29 \%)$ & 21(16.67\%) & $20(26.32 \%)$ \\
\hline$\geq 45$ & $15(6.73 \%)$ & 0 & $11(8.73 \%)$ & $4(5.26 \%)$ \\
\hline \multicolumn{5}{|l|}{ Marriage status } \\
\hline Single & 168(75.34\%) & 16(76.19\%) & $93(73.81 \%)$ & 59(77.63\%) \\
\hline Married & $48(21.52 \%)$ & $4(19.05 \%)$ & $30(23.81 \%)$ & 14(18.42\%) \\
\hline $\begin{array}{l}\text { Divorced/ } \\
\text { widowed }\end{array}$ & $7(3.14 \%)$ & $1(4.76)$ & $3(2.38 \%)$ & $3(3.95 \%)$ \\
\hline \multicolumn{5}{|l|}{ Education } \\
\hline $\begin{array}{l}\text { Middle school } \\
\text { and below }\end{array}$ & $26(11.66 \%)$ & $3(14.29 \%)$ & 15(11.90\%) & $8(10.53 \%)$ \\
\hline High school & $42(18.83 \%)$ & $4(19.05 \%)$ & $28(22.22 \%)$ & $10(13.16 \%)$ \\
\hline $\begin{array}{l}\text { College level } \\
\text { and above }\end{array}$ & $155(69.51 \%)$ & $14(66.67 \%)$ & $83(65.87 \%)$ & $58(76.32 \%)$ \\
\hline
\end{tabular}



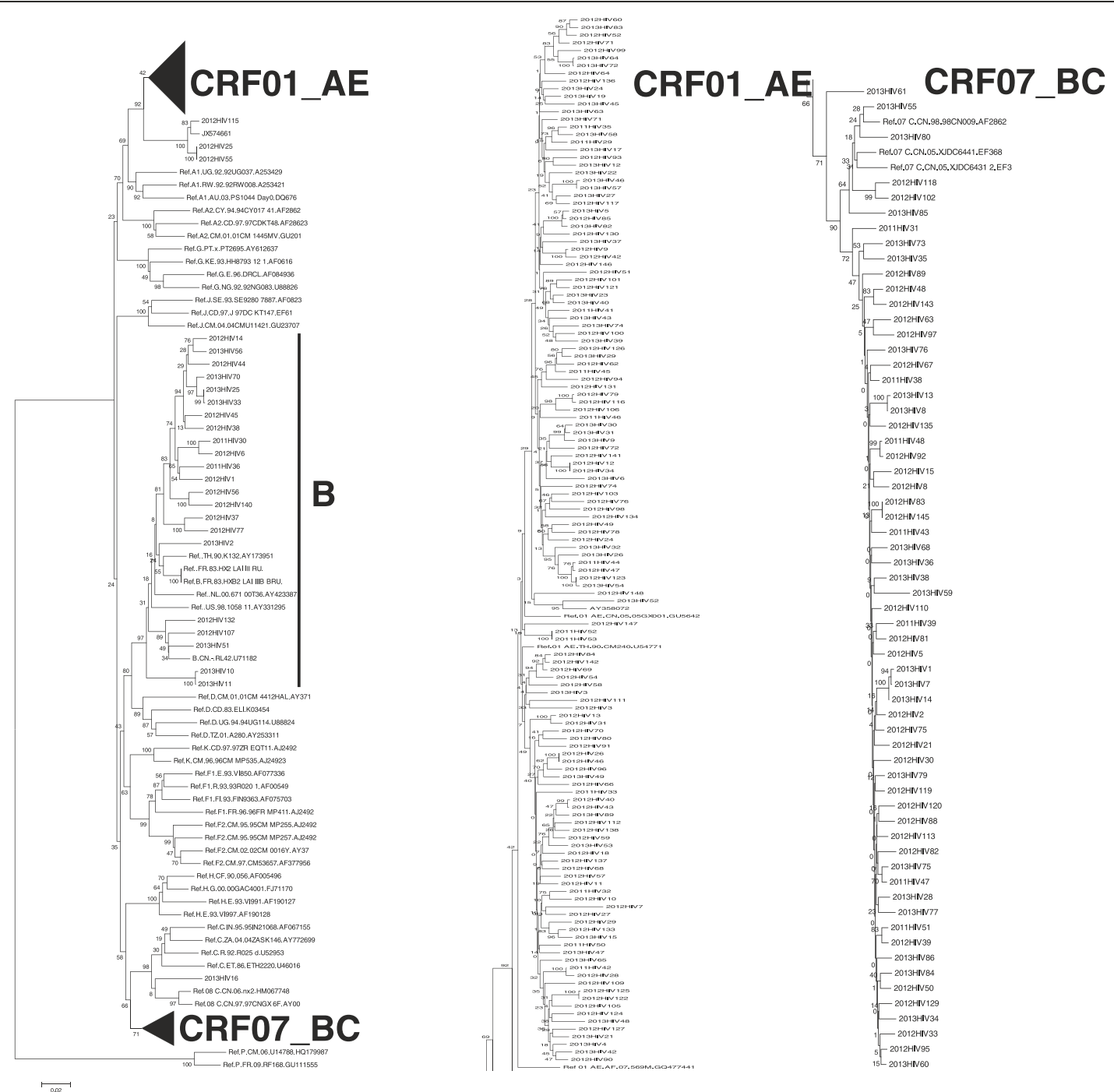

Figure 1 Phylogenetic trees of HIV-1 pol genes were constructed using MEGA 5 based on neighbour-joining methods. The samples' sequences and reference HIV-1 subtypes (subtypes A-D, F-H, J, K, CRF01_AE, CRF07_BC, CRF08_BC, and group O) available in the Los Alamos database were aligned using CLUSTAL W with minor manual adjustments. The statistical robustness of the neighbour-joining tree and reliability of the branching patterns were confirmed by bootstrapping (1000 replicates). The trees were midpoint rooted. Values on the branches represent the percentage of 500 bootstrap replicates.

\section{Prevalence of transmitted drug resistant variants}

Amplified gene regions were assessed for TDR-associated mutations through the Stanford HIV Drug Resistance Database. Mutations were classified and summarized according to their ability of conferring resistance to PI, NRTI, or NNRTI drug classes. Among our samples, 25.6\% (57/223) had TDR-associated mutations, including 16.1\% (36/223) for PI mutations, $0.9 \%$ (2/223) for NRTI mutations, and 9.0\% (20/223) for NNRTI mutations. PI mutations included L10I/V $(7.2 \%, 16 / 223)$ and $\mathrm{A} 71 \mathrm{~L} / \mathrm{T} / \mathrm{V}$ $(6.3 \%, 14 / 223)$, with relatively high frequency; NRTI mutations, included L74I $(0.45 \%, 1 / 223)$ and V75L $(0.45 \%$, $1 / 223)$; and the most frequent NNRTI mutation was V179D/E (5.4\%, 12/223). Among these detected mutations, only L74I, M46L, G190E and E138G may result in drug resistance directly. Most of these mutations only conferred potential drug resistance. The detailed frequency of the mutations was shown in Table 3. Only six (2.7\%) samples carried mutations conferring known levels of drug resistance, with $1.35 \%$ (3/223) against PIs (nelfinavir), $0.45 \%$ (1/223) against NRTIs (abacavir, didanosine), and $0.90 \%$ (2/223) against NNRTIs (efavirenz, etravirine, nevirapine, riplivirine).

The type and frequency of TDR-associated mutations were different among different HIV-1 subtypes. The proportion of TDR-associated mutations was 23.7\% (32/135) among CRF01_AE recombinant strains, the most frequent mutations being L10I/V and V179D/E. Among CRF07_BC 
Table 3 HIV TDR-associated mutations identified among different subtypes/year in treatment-naïve Beijing MSM

\begin{tabular}{|c|c|c|c|c|c|c|c|}
\hline & PI & PI mutations & NRTI & NRTI mutations & NNRTI & NNRTI mutations & Total \\
\hline \multicolumn{8}{|l|}{ Subtype } \\
\hline CRF01_AE & $14.07 \%(19 / 135)$ & $\begin{array}{l}\text { L10IN(14/19) V111(2/19) L33F(2/19) } \\
\text { M46L(2/19) A71L(1/19) }\end{array}$ & $1.48 \%(2 / 135)$ & L74I(1/2) V75L(1/2) & $8.15 \%(11 / 135)$ & V106I(1/11) V179D/E(9/11) G190E(1/11) & $23.70 \%(32 / 135)$ \\
\hline CRF07_BC & $19.35 \%(12 / 62)$ & L10I(1/12) L33I(1/12) Q58E(2/12) A71T/N(8/12) & 0 & - & $1.61 \%(1 / 62)$ & $\mathrm{E} 138 \mathrm{G}(1 / 1)$ & $20.97 \%(13 / 62)$ \\
\hline Subtype B & $22.72 \%(5 / 22)$ & L10I(1/5) A71T/N(5/5) & 0 & - & $31.81 \%(7 / 22)$ & V106I(5/7), V179E(2/7) & $54.54 \%(12 / 22)$ \\
\hline \multicolumn{8}{|l|}{ Year } \\
\hline 2011 & $4.77 \%(1 / 21)$ & L10I(1/1) & 0 & - & $9.52 \%(2 / 21)$ & V106I(2/2) & $14.29 \%(3 / 21)$ \\
\hline 2012 & $17.24 \%(22 / 126)$ & $\begin{array}{l}\mathrm{L} 101 / N(8 / 22) \text { V11।(2/22) L33I/F(3/22) } \\
\text { M46L(2/22) Q58E(1/22) A71T/N(6/22) }\end{array}$ & $2.63 \%(2 / 126)$ & $\mathrm{L} 74 \mathrm{I}(1 / 2), \mathrm{V} 75 \mathrm{~L}(1 / 2)$ & $11.1 \%(14 / 126)$ & $\begin{array}{l}\text { V106I(4/14) E138G(1/14) } \\
\text { V179D/E(8/14) G190E(1/14) }\end{array}$ & $29.37 \%(37 / 126)$ \\
\hline 2013 & $17.11 \%(13 / 76)$ & L10IN(5/13) Q58E(1/13) A71T/N(8/22) & 0 & - & $5.26 \%(4 / 76)$ & V179D(4/4) & $22.37 \%(17 / 76)$ \\
\hline Total & $16.14 \%(36 / 223)$ & & $0.89 \%(2 / 223)$ & & $8.52 \%$ (19/223) & & $25.56 \%(57 / 223)$ \\
\hline
\end{tabular}


recombinant strains, $21.0 \%(13 / 62)$ had TDR-associated mutations, A71T/V being the most frequent. Among subtype B, 54.5\% (12/22) had TDR-associated mutations, $\mathrm{A} 71 \mathrm{~T} / \mathrm{V}$ and V106I being the most frequent. Detailed information is summarized in Table 3.

Of note, the distribution of TDR-associated mutations differed by sampling year (Table 3). In 2011, 14.2\% (3/21) had TDR-associated mutations, while the latter two years saw rates that were more than two times as high, with $29.4 \%(37 / 126)$ in 2012 and 22.4\% (17/76) in 2013.

\section{Co-variation between transmitted drug resistance and polymorphisms}

Co-variation analysis was performed to determine mutations or polymorphisms that were positively selected in association with TDR-associated mutations (Table 4). Eight mutation pairs with significant co-variation were identified in the RT region for CRF01_AE strains between the V179D/E TDR-associated mutation and seven overlapping polymorphisms. No significant mutation pair was identified for the PR region and for other subtypes.

\section{Discussion}

In this study, we analyzed the HIV-1 pol gene sequences of 223 ART treatment-naïve MSM in Beijing diagnosed from 2011 to 2013. The genotype CRF01_AE accounted for $60.5 \%$, followed by CRF07_BC (27.8\%), subtype B (9.9\%), and others. Fifty-seven samples had at least one TDR-associated mutation, mainly including L10I/V (6.3\%), A71L/T/V (6.3\%), V179D/E (5.4\%), and V106I (2.7\%), with different distributions of TDR-associated mutations by different HIV-1 subtypes and by sample year. Moreover, eight significant co-variation pairs were found between TDR-associated mutations (V179D/E) and seven overlapping polymorphisms in subtype CRF01_AE. This analysis consists the most comprehensive genetic characterization to date on HIV-1 TDR associated mutations prevalent among MSM in China.

\begin{tabular}{lllll}
\multicolumn{5}{l}{$\begin{array}{l}\text { Table } 4 \text { Co-variation pairs between overlapping } \\
\text { polymorphisms and TDR-associated mutations }\end{array}$} \\
\hline $\begin{array}{l}\text { TDR- } \\
\text { associated } \\
\text { mutations }\end{array}$ & Polymorphisms & $\begin{array}{l}\text { Jaccard } \\
\text { index }\end{array}$ & P value & $\begin{array}{l}\text { P value } \\
\text { (adjusted) }\end{array}$ \\
\hline V179D & R238K & 0.19047619 & 0.003425851 & 0.052072937 \\
V179D & A272P & 0.125 & 0.020837895 & 0.197960003 \\
V179E & T11K & 0.07894737 & 0.020123086 & 0.197960003 \\
V179E & I173K & 0.08333333 & 0.01703163 & 0.197960003 \\
V179E & K174Q & 0.14285714 & 0.003172559 & 0.052072937 \\
V179E & S207Q & 1 & $2.39 E-06$ & 0.000181289 \\
V179E & S211K & 0.42857143 & $8.35 E-05$ & 0.003172559 \\
V179E & R238K & 0.15789474 & 0.002311436 & 0.052072937 \\
\hline
\end{tabular}

The proportion of samples with TDR-associated mutations over the sample period was determined to be $25.6 \%$. The mutations frequency rose from $14.3 \%$ in 2011 to 29.4\% in 2012 and 22.4\% in 2013. In addition, the distribution of mutations sites in 2011 was simpler compared with 2012 and 2013. This indicated that TDR-associated mutations may be becoming increasingly common and complex among Beijing MSM over the last several years. Although most of these mutations only conferred potential drug resistance, TDR-associated mutations often confer disadvantage in replicative fitness to the virus. A high prevalence and increasingly complex patterns of TDRassociated mutations suggests a generally high frequency of exposure in the treatment-naïve patients to diverse sources of viral strains. Thus, TDR-associated mutations in Beijing MSM may serve as evidence for prevalent high risk behavior such as repeated unprotected exposure to multiple sexual partners.

The largest proportion of the HIV-1 strains among our samples belonged to the CRF01_AE genotype (60.5\%), followed by CRF07_BC (27.8\%) and subtype B (9.9\%), and the distribution of HIV-1 subtypes was relatively stable from 2011 to 2013. This differed from previous reports on Beijing MSM from 2005, which saw higher prevalence of subtype B and lower prevalence of CRF01_AE and CRF07_BC [16]. In the study performed by $\mathrm{Li}$ et al. from 2007 to 2010 [17], the proportion of subtype CRF01_AE, $\mathrm{B}$, and CRF07_BC were $56.0 \%, 30.8 \%$, and $12.6 \%$, respectively. Comparing to previous reports, the ratio of subtype $B$ shows a tendency to drop, while the proportions of CRF01_AE and CRF07_BC are continuously on the rise in MSM population in Beijing. Historically, CRF01_AE was strongly associated with sexual transmission routes [23], while CRF07_BC was associated with intravenous drug user populations in China [24,25]. It is likely that the increase in CRF07_BC prevalence among MSM was linked with transmission among drug users [26].

We found unequal distributions of mutations in the different genotypes, with subtype $B$ having a higher rate $(54.5 \%)$ of TDR-associated mutations than in CRF07_BC and CRF01_AE. This observation coincides with results from a study among Thai patients, in which multivariate analysis showed that HIV-1 subtype $B$ had a higher rate of drug resistance-associated mutations [27]. On the other hand, TDR-associated mutations among CRF01_AE strains showed a diversifying trend. Nonetheless, the most frequent mutations L10I/V and V179D/E appeared to be conserved; the latter was found in 9 out of 11 CRF01_AE strains with drug resistance mutations to NNRTI drugs.

The relative prevalence of V179D/E bears further remark. In a previous study, Archer et al. found that V179D (a NNRTI resistance mutation) significantly reduced the replication capacity of HIV-1 [28]. Here, our co-variation 
analysis showed that V179D/E was significantly associated with seven polymorphisms in the HIV-1 CRF01_AE genotype. These polymorphisms may serve to compensate the replication disadvantage of V179D/E, allowing this TDRassociated mutation to be propagated in treatment-naïve patients [5]. In another study by our team, we had applied the CorMut algorithm to investigate the association between drug resistance and compensatory mutations, and demonstrated that K101Q, H221Y, and T139K can enhance K103N/Y181C/G190A-associated NNRTI-resistance among CRF07_BC in vitro [29]. Certainly, further study is needed to determine how the V179D/E-associated polymorphisms affect the replication fitness of CRF01_AE HIV-1 strains.

However, there are some insufficiency still exists in our study. First, the study patients were newly diagnosed, but we can't confirm the time of initial infection. Some TDR-associated mutations have been missing during this period. Another limitation of the study is related to the use of population sequencing strategy. Such a conventional genotyping technique does not allow for the detection of clinically important minority variants with TDR-associated mutations [30]. Despite some limitations, current study may facilitate the tracking of TDR-associated mutations, and should provide a reliable data on prevalence of the TDR-associated mutations among MSM in Beijing.

\section{Conclusions}

Our work comprehensively characterized HIV-1 strains prevalent among treatment-naïve MSM in Beijing from 2011 to 2013, including subtype analysis, TDR-associated mutations, and co-variation of potential compensatory mutations. TDR mutation rate remains low, but the rate of TDR-associated mutations is high. There are significant co-variation pairs between TDR-associated mutations (V179D/E) and seven overlapping polymorphisms among subtype CRF01_AE. These findings enhance our understanding of TDR and evolution of HIV-1 among Chinese MSM, and suggest that high risk behavior patterns that facilitate the transmission of drug resistant HIV strains remain highly prevalent, requiring stronger prevention and control efforts.

\section{Competing interests}

The authors declare that they have no competing interests.

\section{Authors' contributions}

YJ and SL - conceived experimental design, performed laboratory and demographic data analysis, and wrote the manuscript. ZL, QY and YW - performed demographic data analysis. JZ, LL, and ZZ - performed laboratory experiments and analysis. ZZ and LW - conducted patient enrollment and clinical evaluation for the ECMC-US cohort. YS and LM - supervised manuscript writing and data analysis. All authors read and approved the final manuscript.

\section{Acknowledgements}

This work was supported by grants from the State Key Laboratory for Infectious Disease Prevention and Control (2011SKLID102), National Natural Science Foundation of China $(81172733,812611203)$, the 11th Five-Year National Science \& Technology Major Project (2009ZX10004-903-006), and the 12th Five-Year National Science \& Technology Major Project (2012ZX10004904-002-002, 2013ZX10001-006).

\section{Author details}

${ }^{1}$ State Key Laboratory for Infection Disease Prevention and Control, National Center for AIDS/STD Control and Prevention (NCAIDS), Collaborative Innovation Center for Diagnosis and Treatment of Infectious Diseases, Chinese Center for Disease Control and Prevention (China CDC), Beijing 102206, China. ${ }^{2}$ Beijing Chaoyang District Centre for Disease Control and Prevention, Beijing 100021, China.

Received: 22 June 2014 Accepted: 9 December 2014 - 18.020

\section{References}

1. Frentz D, Boucher CA, van de Vijver DA: Temporal changes in the epidemiology of transmission of drug-resistant HIV-1 across the world. AIDS Rev 2012, 14:17-27.

2. Zhang FJ, Pan J, Yu L, Wen Y, Zhao Y: Current progress of China's free ART program. Cell Res 2005, 15:877-882.

3. Yu X, Yuan L, Huang Y, Xu W, Fang Z, Liu S, Shao Y, Jiang S, Ma L: Susceptibility of HIV-1 subtypes B', CRF07_BC and CRF01_AE that are predominantly circulating in China to HIV-1 entry inhibitors. PLoS One 6: e17605.

4. Ceccherini-Silberstein F, Svicher V, Sing T, Artese A, Santoro MM, Forbici F, Bertoli A, Alcaro S, Palamara G, d'rminio Monforte A, Balzarini J, Antinori A, Lengauer T, Perno CF: Characterization and structural analysis of novel mutations in human immunodeficiency virus type 1 reverse transcriptase involved in the regulation of resistance to nonnucleoside inhibitors. J Virol 81:11507-11519.

5. Li Z, Huang Y, Ouyang Y, Xing H, Liao L, Jiang S, Shao Y, Ma L: Mutation covariation of HIV-1 CRF07_BC reverse transcriptase during antiretroviral therapy. J Antimicrob Chemother 2013, 68:2521-2524.

6. Taiwo B: Understanding transmitted HIV resistance through the experience in the USA. Int J Infect Dis 2009, 13:552-559.

7. Koblin BA, Chesney MA, Husnik MJ, Bozeman S, Celum CL, Buchbinder S, Mayer K, McKirnan D, Judson FN, Huang Y, Coates TJ, EXPLORE Study Team: High-risk behavior among men who have sex with men in 6 US cities: baseline data from the EXPLORE study. Am J Public Health 2003, 93:926-932.

8. Liu H, Yang H, Li X, Wang N, Liu H, Wang B, Zhang L, Wang Q, Stanton B: Men who have sex with men and human immunodeficiency virus/Sexually transmitted disease control in China. Sex Transm Dis 2006, 33:68-76.

9. State Council AIDS Working Committee Office (SCAWCO): 2012 China AIDS Response Progress Report, Ministry of Health of the People's Republic of China. China: Annual Report; 2012.

10. Choi KH, Liu H, Guo Y, Han L, Mandel JS, Rutherford GW: Emerging HIV-1 epidemic in China in men who have sex with men. Lancet 2003, 361:2125-2126.

11. Li SW, Zhang XY, Li XX, Wang MJ, Li DL, Ruan YH, Zhang XX, Shao YM: Detection of recent HIV-1 infections among men who have sex with men in Beijing. Chin Med J (Engl) 2008, 121:1105-1108.

12. Beijing Centers for Diseases Control and Prevention (CDC) \&Centers for Preventive Medical Research: 2012 Beijing AIDS Report. Beijing: Annual Report; 2013.

13. Cai $W D$, Feng $T$, Tan JG, Chen L, Shi XD, Chen PL, Jiang LZ, Tao XY: $A$ survey of the characteristics and STD/HIV infection of homosexuality in Shenzhen. Mod Prev Med 2005, 32:328-330.

14. Choi KH, Ning Z, Gregorich SE, Pan QC: The influence of social and sexual networks in the spread of HIV and syphilis among men who have sex with men in Shanghai, China. J Acquir Immune Defic Syndr 2007, 45:77-84.

15. Gu Y, Qu P, Xu L, Luo M, Wang XL, Gu J, Zhao LL, Lu YH, Zhou BS: Survey of knowledge, attitude, behavior and practices related to STI/HIV among male homosexuality in Shenyang. Chin Pub Health 2004, 20:573-574.

16. Zhang $X Y$, Wang $C$, Li XX, Zhang XX, Song YH, Li SW, Ma LY, Shi W, Xu JQ, Yang ZK, Ruan YH, Hong KX, Xing H, Shao YM: Study of HIV infection coinfection with STDs and HCV and related changes in immunological 
indicators and viral loads among men who have sex with men in Beijing. Chin J AIDS STD 2006, 12:294-296.

17. Li L, Han N, Lu J, Li T, Zhong X, Wu H, Rayner S, Chen L, Liu Y, Wang X, Li H, Li J: Genetic characterization and transmitted drug resistance of the HIV type 1 epidemic in men who have sex with men in Beijing, China. AIDS Res Hum Retroviruses 2013, 29:633-637.

18. Liao L, Xing H, Li X, Ruan Y, Zhang Y, Qin G, Shao Y: Genotypic analysis of the protease and reverse transcriptase of HIV type 1 isolates from recently infected injecting drug users in western China. AIDS Res Hum Retroviruses 2007, 23:1062-1065.

19. Tamura K, Peterson D, Peterson N, Stecher G, Nei M, Kumar S: MEGA5: molecular evolutionary genetics analysis using maximum likelihood, evolutionary distance, and maximum parsimony methods. Mol Biol Evol 2011, 28:2731-2739

20. Li Z, Huang $Y$, Ouyang $Y$, Jiao $Y$, Xing $H$, Liao L, Jiang $S$, Shao $Y$, Ma L: CorMut: an R/Bioconductor package for computing correlated mutations based on selection pressure. Bioinformatics 2014, 30:2073-2075.

21. Chen L, Perlina A, Lee CJ: Positive selection detection in 40,000 human immunodeficiency virus (HIV) type 1 sequences automatically identifies drug resistance and positive fitness mutations in HIV protease and reverse transcriptase. J Virol 2004, 78:3722-3732.

22. Rhee SY, Liu TF, Holmes SP, Shafer RW: HIV-1 subtype B protease and reverse transcriptase amino acid covariation. PLoS Comput Biol 2007, 3:e87.

23. Lin H, He N, Zhou S, Ding Y, Qiu D, Zhang T, Wong FY: Behavioral and molecular tracing of risky sexual contacts in a sample of Chinese HIVinfected men who have sex with men. Am J Epidemiol 2013, 177:343-350.

24. Lu L, Jia M, Ma Y, Yang $L$, Chen $Z$, Ho DD, Jiang $Y$, Zhang $L$ : The changing face of HIV in China. Nature 2008, 455:609-611.

25. Liao L, Xing H, Shang H, Li J, Zhong P, Kang L, Cheng H, Si X, Jiang S, Li X, Shao $Y$ : The prevalence of transmitted antiretroviral drug resistance in treatment-naïve HIV-infected individuals in China. J Acquir Immune Defic Syndr 2010, 53:S10-S14.

26. Tee KK, Pybus OG, Li XJ, Han X, Shang H, Kamarulzaman A, Takebe Y: Temporal and spatial dynamics of human immunodeficiency virus type 1 circulating recombinant forms 08 BC and 07 BC in Asia. J Virol 2008, 82:9206-9215

27. Manosuthi W, Thongyen S, Nilkamhang S, Manosuthi S, Sungkanuparph S: HIV-1 drug resistance-associated mutations among antiretroviral-naïve Thai patients with chronic HIV-1 infection. J Med Virol 2013, 85:194-199.

28. Archer RH, Dykes C, Gerondelis P, Lloyd A, Fay P, Reichman RC, Bambara RA, Demeter LM: Mutants of human immunodeficiency virus type 1 (HIV-1) reverse transcriptase resistant to nonnucleoside reverse transcriptase inhibitors demonstrate altered rates of RNase $\mathrm{H}$ cleavage that correlate with HIV-1 replication fitness in cell culture. J Viro/ 2000, 4:8390-8401.

29. Huang $Y$, Li Z, Xing H, Jiao Y, Ouyang $Y$, Liao L, Jiang $S$, Armstrong $R$, Shao $Y, M a$ L: Identification of the critical sites of NNRTI-resistance in reverse transcriptase of HIV-1 CRF_BC strains. PLoS One 2014, 9:e93804.

30. Simen BB, Simons JF, Hullsiek KH, Novak RM, Macarthur RD, Baxter JD, Huang C, Lubeski C, Turenchalk GS, Braverman MS, Desany B, Rothberg JM Egholm M, Kozal MJ: Terry Beirn community programs for clinical research on AIDS: Low-abundance drug resistant viral variants in chronically HIV-infected, antiretroviral treatment-naive patients significantly impact treatment outcomes. J Infect Dis 2009, 199:693-701.

doi:10.1186/s12879-014-0689-7

Cite this article as: Jiao et al:: HIV-1 transmitted drug resistance-associated mutations and mutation co-variation in HIV-1 treatment-naïve MSM from 2011 to 2013 in Beijing, China. BMC Infectious Diseases 2014 14:689.

\section{Submit your next manuscript to BioMed Central and take full advantage of:}

- Convenient online submission

- Thorough peer review

- No space constraints or color figure charges

- Immediate publication on acceptance

- Inclusion in PubMed, CAS, Scopus and Google Scholar

- Research which is freely available for redistribution

Submit your manuscript at www.biomedcentral.com/submit
Ciomed Central 